\title{
Towards a unified structure for AGN and accretion powered sources
}

\section{Demosthenes Kazanas*}

Astrophysics Science Division, NASA/GSFC, Greenbelt, MD 20771

E-mail: demos.kazanasdnasa.gov

\section{Keigo Fukumura}

Physics Department, James Mason University, Harrisonburg, VA

E-mail: fukumukxejmu.edu

\section{Ehud Behar}

Department of Physics, Technion, Haifa, Israel

E-mail: behardphysics.technion.ac.in

\section{Ioannis Contopoulos}

Academy of Athens, Soranou Efesiou 4, Athens, Greece

E-mail: icontopdacademyofathens.gr

We propose that the ensemble of AGN X-ray absorber data can be accounted for in terms of MHD winds launched off their accretion disks. We note in particular, that the winds that fit the absorption features of the Chandra and XMM Seyfert spectra ought to have density profiles $\propto 1 / r$. Our models can produce 2D winds with the required density profiles and a very steep dependence in the polar angle $\theta$; as such they can naturally serve as the "tori" required by the AGN unification. The winds and absorption spectra of our models depend on the dimensionless mass flux rate, $\dot{m}$, the disk inclination angle $\theta$ and the X-ray content of the spectra, given by the parameter $\alpha_{\mathrm{OX}}$. However, a relation between $\alpha_{\mathrm{OX}}$ and the AGN luminosity reduces the number of parameters to two. The same dynamical models can account also for the X-ray absorber properties of galactic $\mathrm{X}$-ray Binaries (XRB), once the proper contribution of X-rays to the ionizing spectrum has been taken into account.

An INTEGRAL view of the high-energy sky (the first 10 years)" 9th INTEGRAL Workshop and celebration of the 10th anniversary of the launch,

October 15-19, 2012

Bibliotheque Nationale de France, Paris, France

\footnotetext{
* Speaker.
} 


\section{Introduction}

The launch of Chandra and XMM-Newton ushered a new era in X-ray astronomy of AGN outflows with the discovery of absorption lines in the spectra that enabled for the first time accurate charge state and velocity measurements. The long observations of a number of AGNs revealed Xray transitions of charge states as diverse as Fe I through Fe XXVI. This underscores the utility of $\mathrm{X}$-ray spectroscopy which within $\gtrsim 1$ decade in energy encompasses transitions from ionic species of plasmas that span $\sim 5$ decades in ionization parameter $\xi$. Similar progress was achieved in the UV study of AGN winds with the launch of HST, which showed that $\sim 50 \%$ of AGN show evidenced of outflow absorption in their spectra, similar in percentage with those that show X-ray absorption; apparently there is a relation between the X-ray and UV absorption feature properties, which however it is not quite as clear yet [ 6 ].

The large number and broad ionization parameter range of the X-ray transitions in the Chandra and XMM spectra allows for statistical treatments. Thus, [Q] and [W] assumed a continuous distribution of absorbers with $\xi$ and using an error minimization procedure for the entire set of transitions, computed the distribution of their hydrogen-equivalent column $N_{H}$ as a function of $\xi$; this way they produced what they called the absorption measure distribution (AMD), namely the differential hydrogen-equivalent column $N_{H}$ of specific ions per decade of $\xi$, i.e. AMD = $d N_{H} / d \log \xi$. Most importantly, they found that the AMD has a rather weak dependence on $\xi$; in the small number of Seyferts for which the data quality allowed a quantitative analysis they found $d N_{H} / d \log \xi \propto \xi^{p}, 0 \lesssim p \lesssim 0.3$. The functional form of the AMD is significant as it can provide the plasma density along the observer's line of sight (LoS); one can easily see that AMD $=d N_{H} / d \log \xi \propto \xi^{p} \propto N_{H} \propto \xi^{p}$, implies $n(r) \propto r^{-\alpha}$, with $\alpha=(2 p+1) /(p+1)$; so the observed range in $p$, limits $\alpha$ to the range $1 \lesssim \alpha \lesssim 1$. This finding is significant because the corresponding density profile is inconsistent with the $n(r) \propto r^{-2}$ density profile of radiation driven winds, the preferred means of launching winds in AGN and compact objects in general. This fact alone implies that these winds are likely driven by the action of magnetic fields, i.e. that they are the MHD outflows. In addition, the broad range in the values of ionization parameter $\xi$ of the observed transitions, implies that these winds extend over a similar range in radius; thus the $\mathrm{X}$-ray absorber spectroscopy affords a global view of the structure of AGN along the observer's LoS, exclusive to this energy band.

The formal analysis of 2D MHD winds in relation to AGN was enunciated by [B]]; they produced 2D solutions of the steady state, axisymmetric MHD equations involving a poloidal magnetic field threading a thin, rotating Keplerian accretion disk. As such, they extend over the entire radial range of the disk, from near the Schwarzschild radius $R_{S}$ to its outer edge, believed to be at a distance of $10^{6} R_{S} \sim 1-10$ parsec in AGN. Therefore, they provide structures of radial range consistent with that of the ionization parameter of X-ray absorber features; as such, these winds are reasonable candidates for the plasma responsible for these absorption features.

The winds studied in [3] have a density profile $n(r) \propto r^{-3 / 2}$, inconsistent with that inferred from the AMD. Generalized version of these winds that allow for a range of the exponent $\alpha$ consistent with observations were found and studied by [[]]. Furthermore, winds similar to those of []] with $n(r) \propto r^{-1}$ were invoked by [12] as the "torii" of AGN unification, as winds with this density profile provided, by reprocessing of the AGN UV radiation, the best fit to their IR spectra. This 

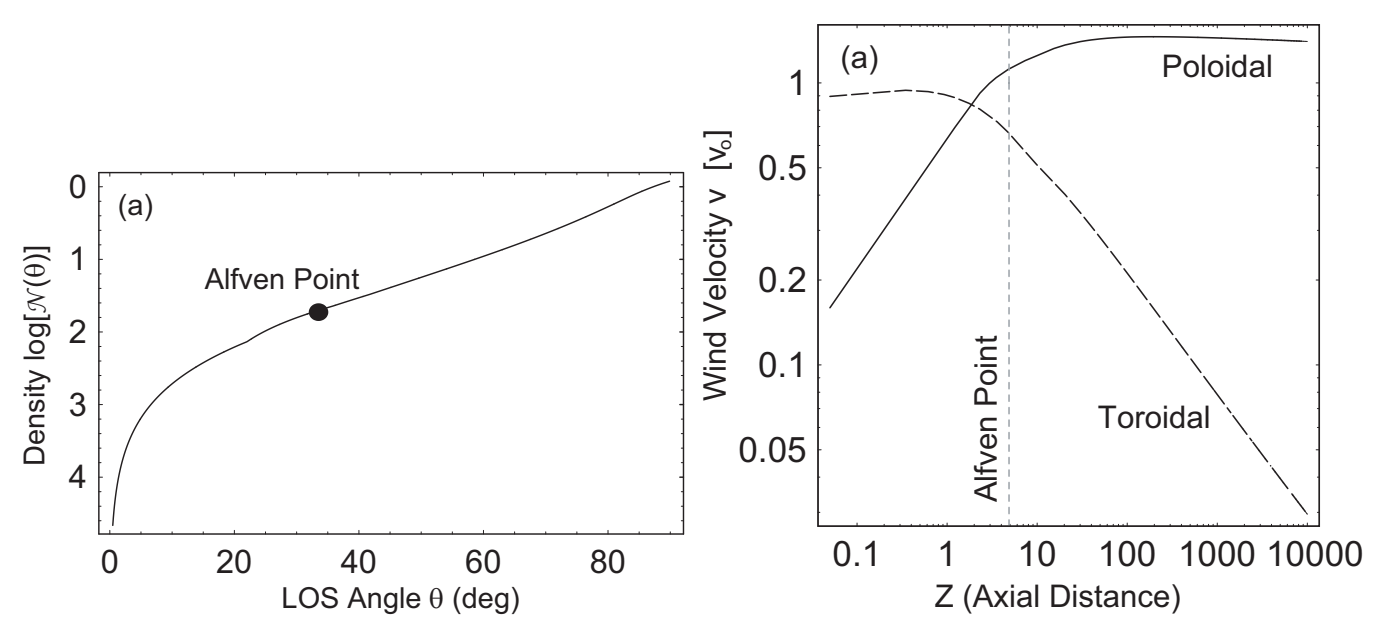

Figure 1: (a-left) The angular distribution of wind density in the poloidal plane normalized to its value at $\theta=90^{\circ}$. (b-right) The poloidal and toroidal wind velocities as a function of height above the disk normalized to the Keplerian velocity on the disk plane.

work, by suggesting the AGN torii to be dynamical (rather than in hydrostatic equilibrium) objects, resolved the conundrum of requiring (by AGN statistics) these torii to be thick, i.e. $H / R \simeq 1$ ( $H$ is with torus height) while the thermal velocities (they have temperatures $T \sim 10-100 \mathrm{~K}$ ) require $H / R \lesssim 10^{-3}$. Along the same lines, Rowan-Robinson [ए]] showed that one can reproduce the IRFIR spectra of bright quasars by dust reprocessing their UV-X-ray continua by dust that extends over several decades in radius. He showed then that the best fit of these spectra require a radial dependence of the dust density $n_{d}(r) \propto r^{-1}$.

There is therefore mounting evidence that the AGN central black holes are surrounded, over several decades in radius, by the plasma of MHD winds emanating from their accretion disks. While roughly axisymmetric, these winds have a rather unique density distribution, in that it drops like $\simeq 1 / r$ in the radial direction, while it has a sufficiently steep $\theta$-dependence to provide the obscuration needed to account for the Seyfert 1 - Seyfert 2 unification on the basis of the observer's LoS direction (see fig. 1a). The specific radial density dependence is very important, as it implies roughly equal column per decade of radius, a fact that makes possible the detection of ionic species as diverse as Fe I and Fe XXV in the same object. Much steeper density profiles would make the low ionization ionic species all but impossible to detect, while much flatter than the above would lead to much higher columns for the low $\xi$ ions than observed and would likely provide IR-tofar-IR fits inconsistent with observation. Motivated by these considerations we have embarked in the detailed study of ionization of MHD winds and their entailing observational consequences. In this talk we focus on the broader scaling properties of these winds and their broader role in understanding AGN unification.

\section{The MHD Wind Structure and Ionization}

The MHD winds we have in mind are by construction 2 dimensional objects. As such the equations describing them are partial differential equations. To make progress, separation of variables is of the essence. Blandford \& Payne [B] achieved this demanding self-similarity of the poloidal 
field lines, i.e. that they are all versions of a given line (which crosses the equatorial plane at radius $r_{o}$ ) homothetically displaced along the radial direction (i.e. they all make the same angle that any radial vector makes with the sample line); the radius $r_{o}$ is then considered as the unit to which all radial distances are compared to. This allows the separation of variables and after considering all integrals of motion, one is left with the force balance equation in the $\theta$-direction, the so called Grad-Safranov equation. This has the character of a wind equation and a solution requires one to cross the Alfvén and fast magnetosonic points before asymptotic velocity is achieved. One should note here that the condition of self-similarity, while absolutely necessary for the separation of variables, is a reasonable assumption for assessing the overall properties of structures that span 5-8 decades in radius, else these solutions would make no sense observationally.

With these conventions, the magnetic field, velocity, pressure and density of the wind are given by

$$
\begin{aligned}
\mathbf{B}(r, \theta) & \equiv x^{-(s+1) / 2} \tilde{\mathbf{B}}(\theta) B_{o} \\
b f v(r, \theta) & \equiv x^{-1 / 2} \tilde{\mathbf{v}}(\theta) v_{o} \\
p(r, \theta) & \equiv x^{-(s+1)} \mathscr{P}(\theta) B_{o}^{2} \\
n(r, \theta) & \equiv x^{-s} \tilde{n}(\theta) B_{o}^{2} v_{o}^{-2}
\end{aligned}
$$

where $x=r / r_{o}$ is the normalized radial distance. The density normalization is given in terms of the poloidal field on the disk $B_{o}$, however, it is more instructive to express it in terms of the accretion or wind outflow rate $\dot{m}$ as discussed in [D]; then the density normalization at the inner edge of the disk at $\theta=90^{\circ}$ is given by [setting $\tilde{n}\left(90^{\circ}\right)=1$ and the $\theta$-dependence $\tilde{n}$ given by Fig. 2a of [四]

$$
n_{o}=\frac{f_{W} \dot{m}_{o}}{2 \sigma_{T} r_{s}}
$$

where $f_{W}$ is the ratio of of the mass-outflow rate in the wind to the mass-accretion rate $\dot{m}_{o}$, assumed here to be $f_{W} \simeq 1$, and $\sigma_{T}$ is the Thomson cross-section. It is important to note here that because the mass flux in these winds depends in general on the radius, the normalized parameter used throughout this work, $\dot{m}_{0}$, always refers to the mass flux at the innermost value of the flow radius, i.e. at $x \simeq 1$. With the scalings given above we then have $\dot{m}(x)=\dot{m}_{o} x^{-s+3 / 2}$, so for $s=1, \dot{m} \propto x^{1 / 2}$ (in the []] solution, $s=3 / 2$ and $\dot{m}=$ const.).

The increasing mass flux with radius sounds at first time counterintuitive. We urge the reader to look at the article of [D], where it is explained that the increase in $\dot{m}$ with radius is due to the necessity of the accretion flow to rid of the excess angular momentum and energy transferred outward by the viscous torques. There is evidence that this is also true in galactic sources [45]].

With the density profile given, one can now compute the ionization structure of the wind. Details and examples of this procedure are given in [0, [8]. Here we present only the scaling relations. The wind ionization is determined by the local ratio of photons to electrons, the ionization parameter $\xi=L / n(r) r^{2}$, ( $L$ is the source's ionizing luminosity, $n(r)$ the local density and $r$ the distance from the ionizing source). This can also be expressed in dimensionless units: If $\eta(\simeq 10 \%)$ is the radiative efficiency of the accretion process, then the luminosity $L$ can be written as $L \propto \eta \dot{m}_{a} M\left(\dot{m}_{a}\right.$ is the accretion rate that reaches the compact object to produce the luminosity $L$ ), or $L \propto \eta \dot{m}_{a}^{2} M$ in the case of ADAF [14]] [i.e for $\dot{m}_{a} \lesssim \alpha^{2}$ with $\alpha$ the disk viscosity parameter], yielding the for $\xi$ 
an expression also independent of $M$, implying similarity in wind ionization, whether in AGN or XRB [प, प]

$$
\xi(x) \simeq \frac{L}{n(r) r^{2}} \simeq \frac{\eta \dot{m}_{a}}{N_{H}(x) x} \simeq \begin{cases}10^{8} \frac{\eta}{f_{W}} \frac{1}{x^{-s+2}} & \text { for } \dot{m}_{a}>\alpha^{2}(\text { non-ADAF }) \\ 10^{8} \frac{\eta}{f_{W}} \frac{\dot{m}_{a}}{x^{-s+2}} & \text { for } \dot{m}_{a}<\alpha^{2}(\mathrm{ADAF})\end{cases}
$$

where $f_{w}=\dot{m}_{w 0} / \dot{m}_{a}(\sim 1)$ is the ratio of mass flux in wind and accretion at the smallest radii and $p$ the parameter of $\dot{m}$ dependence on $r$.

Writing Eq. (12.6) as $N_{H}(x) \propto \eta \dot{m}_{a} / \xi(x) x$ one can form the expression for AMD (HBK07), namely

$$
A M D=\frac{d N_{H}(x)}{d \log \xi(x)} \simeq \frac{\eta \dot{m}_{a}}{\xi(x) x}
$$

The fact that AMD is largely independent of $\xi(x)$ (HBK07) implies $\xi(x) \propto 1 / x$ or $N_{H}(x) \propto \log (x)$, $n(x) \propto 1 / x$ and $\dot{m} \propto x^{1 / 2}$, i.e. the wind mass flux increases with radius, as discussed above. Both the ionization parameter and the wind density decrease like $1 / r$ while the column of the ions found at a given $\xi$ remains roughly constant, in broad agreement with [四]. So, for sufficiently large accretion disks, the wind launched in their largest radii will be sufficiently cool to conform with the properties of the AGN unification torus [12], including the angular distribution of the gas column along the observer's LoS (see fig. 1a). For face-on AGN the winds are highly ionized and should exhibit little absorption (This is apparently the case with Mkn 509; see [ए]]).

As indicated by Eq.(2.6) above, matter at small $x$ is highly ionized, so the first transitions should occur at distances with $\xi \lesssim 10^{4}$, i.e. at distances $x \gtrsim 10^{4}$ or velocities $v \sim 10^{-2} c \sim 3,000$ $\mathrm{km} / \mathrm{s}$. However, the wind ionization depends in addition on the luminosity of the ionizing radiation; this is determined by the amount of X-ray photons per electron, a quantity measured by $\alpha_{\mathrm{OX}}$, the logarithmic slope of the X-ray to UV fluxes, that apparently varies with the luminosity of sources [ㅁ] . Lower $\alpha_{\mathrm{OX}}$ implies transition to non-fully ionized plasma at much smaller radii and higher velocities for the Fe XXVI, Fe XXV transitions.

The extreme objects in this category are the BAL QSOs with $\alpha_{\mathrm{OX}} \sim-2.0$. Indeed, in the BAL QSO APM 08279+5255 the Fe XXV and C IV absorption features were detected at velocities $v(\mathrm{Fexxv}) \simeq 0.6 c$ [四] and $v(\mathrm{Civ}) \simeq 0.1 c$ respectively [[प]. [ [ $]$ ] have used the MHD wind models discussed herein to model the absorption properties of this object. The low X-ray fraction of this object, indicates that even very close to the continuum source, the plasma is not fully ionized, resulting in the observed high velocity for Fe XXV. At the same time, the now opaque plasma, absorbs and reduces the continuum photons that produce the $\mathrm{C} I \mathrm{~V}$ ion, so the latter also occurs at quite high velocity $(v(\mathrm{Civ}) \simeq 0.1 c)$. At the other end of $\mathrm{X}$-ray dominance, the galactic black hole candidate GRO 1655-40 has exhibited transitions similar to those of AGN at similar columns [[ए3] but much lower velocities ( $v \simeq 1,200 \mathrm{~km} / \mathrm{s}$ for Fe XXV, and $v \simeq 200-400 \mathrm{~km} / \mathrm{s}$ for lower ionization species). This is consistent with the basic premise of our model, namely that all accreting black holes involve in essence the same accretion disk winds with velocities approaching $c$ for radii close to the horizon and fully ionized plasma. However, their appearance (velocity of ions) depends on the distance from the black hole at which the wind plasma ceases to be fully ionized. This distance is larger the larger the X-ray content of the ionizing spectrum. 
Finally, some discussion on the shortcomings of these models. They clearly ignore the effects of radiation pressure. As shown in [ए]] and [ए]], these can be quite important, especially for driving the outflows in BAL QSO. While these are clearly important locally, radiatively driven winds will eventually lead to densities $\propto r^{-2}$ and will lead to extremely low columns for the low ionization ions. One should further note that the UV flux driven BAL QSO winds require shielding the wind gas from the QSO X-rays, since highly ionized gas is transparent to the UV photons. However, the high Fe XXV and C IV velocities of BAL QSO APM 08279+5255 suggest that such shielding is not necessary. Eventually, novel insights on these issues should await the Astro $\mathrm{H}$ mission.

\section{References}

[1] Behar, E., 2009, ApJ, 703, 1346

[2] Blandford, R. R \& Begelman, M. C. 1999, MNRAS, 303, L1 (BB99)

[3] Blandford, R. D. \& Payne, D. G. 1982, MNRAS, 199, 883 (BP82)

[4] Chartas, G., Brandt, W. N., Gallagher, S. C. 2003, ApJ, 595, 85

[5] Contopoulos, J., \& Lovelace, R. V. E. 1994, ApJ, 429, 139 (CL94)

[6] Crenshaw, D. M., Kraemer, S. B. \& George, I. M. 2003, ARA\&A, 41, 117

[7] Fukumura, K., \& Kazanas, D., Contopoulos, I., \& Behar, E. 2010a, ApJ, 715, 636 (FKCB10)

[8] Fukumura, K., \& Kazanas, D., Contopoulos, I., \& Behar, E. 2010b, ApJ, 723, L228

[9] Holczer, T., Behar, E., \& Kaspi, S. 2007, ApJ, 663, 799

[10] Kaastra, J. S.et al. 2012, A\&A, 539, 117

[11] Kazanas, D. et al. Astronomical Review, 7, 92 (arXiv:1206.5022)

[12] Königl, A. \& Kartje, J. F. 1994, ApJ, 434, 446 (KK94)

[13] Miller, J. M. et al. 2008, ApJ, 680, 1359

[14] Narayan, R. \& Yi, 1994, ApJ, 428, L13

[15] Neilsen, J., Remillard, R. A. \& Lee, J. C. 2011, ApJ, 737, 69

[16] Proga, D., Stone, J. M., \& Kallman, T. R. 2000, ApJ, 543, 686

[17] Proga, D. 2003, ApJ, 585, 406

[18] Rowan-Robinson, M. 1995, MNRAS, 272, 737

[19] Srianand, R. \& Petitjean, P. 2000, A\&A, 357, 414

[20] Steffen, A. T. et al. 2006, AJ, 131, 2826 